\title{
Colour particle in confining environment
}

\author{
Viatcheslav Kuvshinov* \\ Joint Institute for Power and Nuclear Research - Sosny of the National Academy of Sciences of \\ Belarus, Minsk (JIPNR - Sosny, Minsk) \\ E-mail: v.kuvshinov@sosny.bas-net.by
}

\section{Eugene Bagashov}

Joint Institute for Power and Nuclear Research - Sosny of the National Academy of Sciences of Belarus, Minsk (JIPNR - Sosny, Minsk)

E-mail: paladin17@yandex.ru

It is shown that a colour particle (spinless heavy quark) moving in a confining environment undergoes the process of decoherence which results in the relaxation of quantum superpositions and the emergence of a mixed quantum state with equal probabilities for different colours. In the multiparticle case this also leads to an emergence of entangled states.

The environment is being modeled on the basis of the stochastic QCD vacuum model, which is briefly described. Decoherence rate is being analysed using the Wilson loop and quantum optics parameters: fidelity, von Neumann entropy, purity. Those are being calculated and the decoherence rate is found to be proportional to the distance between colour charges.

A point is made that the physical reason for this process is the chaotic dynamics of Yang-MillsHiggs fields. Critical energy of order-chaos transition is obtained which depends on Higgs boson mass. Stochasticity may be the root of color confinement: disappearing of color at confinement distances.

XXII International Baldin Seminar on High Energy Physics Problems

15-20 September, 2014

JINR, Dubna, Russia

${ }^{*}$ Speaker. 


\section{Introduction}

The confinement of quarks and gluons can be considered the main problem in the field of hadron matter physics. There is a substantial difficulty in acquiring the analytical solution to this problem, which is caused by the fact that it is impossible to calculate the vacuum averages of gauge-invariant quantities in QCD in a closed form, e.g. using the path integrals. The quarks and gluons do not appear in free states on distance scales more than the size of single hadron. Yet over the years several approaches to mathematical description of confinement were developed, with the use of various models.

The model of stochastic QCD vacuum [1, 2, 3, 4] provides an efficient way of obtaining such a description. This is a nonperturbative, phenomenological approach, which is based on the approximation of nonperturbative $\mathrm{QCD}$ as a Gaussian process. It assumes that one can derive various quantities, such as cross sections or other expectation values of gauge-invariant quantities as expectation values with respect to some well-behaved stochastic term in the Hamiltonian. Here only the second-order cumulants are considered to be non-zero, as opposed to the coherent vacuum, where all the cumulants are important [4]. The main result of this is that the confining effect is provided, giving rise to QCD strings with constant tension at large distances [4, 5]. These strings effectively prevent the quarks from separating infinitely far from each other, attracting forces being equal to the string tension. As a result only white, colourless objects can be observed in free state. Most frequently the model of QCD stochastic vacuum is used to calculate Wilson loops, string tensions and field cofigurations around static charges.

The QCD vacuum can be considered as the environment for colour quantum particles $[6,7$, $8,9,10]$. Interactions of quantum system with the environment can be effectively represented by additional stochastic terms in the Hamiltonian of the system [5]. The resulting density matrix of the system is obtained by averaging with respect to environment [5, 11, 12, 13, 14]. Such interactions with the environment result in decoherence of the initial quantum state and relaxation of quantum superpositions. Thus, instead of considering complicated nonperturbative dynamics of Yang-Mills fields one introduces external stochastic field and average over its implementations. Information on the initial state of the quantum system is lost after the averaging is done, and the system becomes effectively colourless. It is shown that colourless objects can be obtained as mixtures of states described by the density matrix as a result of its initial state evolution. We also address the issues of the order-chaos transition in QCD vacuum in terms of critical energy.

\section{Evolution of colour superposition in QCD vacuum}

Consider a system consisting of an arbitrary colour superposition (which corresponds to the quark wave function) and the surrounding environment, which represents a QCD vacuum. The generalized initial state of the mentioned superposition can be written as:

$$
\left|\phi_{i n}\right\rangle=\alpha|1\rangle+\beta|2\rangle+\gamma|3\rangle
$$

where the ket vectors are the pure states, corresponding to all possible colours in fundamental representation of the $\mathrm{SU}(3)$ gauge group, and the complex valued greek letter coefficients obey the normalization condition $|\alpha|^{2}+|\beta|^{2}+|\gamma|^{2}=1$. It is worth mentioning that the other factors of quark 
state vector (the spin, electric charge etc.) are neglected here. We are considering only properties of the strong interaction itself.

The state (2.1) is pure (since the states $|1\rangle,|2\rangle,|3\rangle$ are pure), and the corresponding density matrix is going to contain non-diagonal terms (correlations):

$$
\hat{\rho}_{\text {in }}=\left|\phi_{\text {in }}\right\rangle\left\langle\phi_{\text {in }}\right|
$$

which in the basis represented by vectors $|1\rangle,|2\rangle,|3\rangle$ can be written as

$$
\hat{\rho}_{i n}=\left(\begin{array}{ccc}
|\alpha|^{2} & \alpha \beta^{*} & \alpha \gamma^{*} \\
\alpha^{*} \beta & |\beta|^{2} & \beta \gamma^{*} \\
\alpha^{*} \gamma & \beta^{*} \gamma & |\gamma|^{2}
\end{array}\right)
$$

The existing non-diagonal components (often referred to as coherences) represent the phase relations (interference) between the states $|1\rangle,|2\rangle,|3\rangle$.

Obviously the normalization condition also holds true here: $\operatorname{Tr}\left(\hat{\rho}_{\text {in }}\right)=1$.

Assume that this quark propagates along some fixed path $M$ from point $x$ to point $y$. As it is shown in $[2,3,4]$, the amplitude of such process in Feynman-Schwinger (proper-time) representation can be found with the use of a parallel transport operator. In the model of QCD stochastic vacuum only expectation values of gauge-invariant quantities are defined [1, 2], and this means that the path-ordering exponents should also be taken over the closed path, so we will consider $x$ $=y$, thus instead of an arbitrary $M$ having a closed path (a loop) $L$. Physically this means that the particle-antiparticle pair is created, propagate and finally annihilate [4].

After doing some calculations (which may be found in the paper [9]), including averaging a derived solution over the implementations of stochastic vacuum, we can write down the resulting density matrix of the system after its transit along the loop:

$$
\hat{\rho}(y)=N_{c}^{-1} \hat{I}+\left(\hat{\rho}_{i n}-N_{c}^{-1} \hat{I}\right) W_{a d j}(L)
$$

where $W_{a d j}(L)$ is the Wilson loop in the adjoint representation [15], $\hat{I}$ is the unit operator and $N_{c}$ is the number of colours.

Wilson loop decays exponentially with the total area spanned in the stochastic vacuum $[3,4$, $5,7,8$ ], thus if we choose a rectangular loop $L$ spanning over time $T$ and distance $R$, we will have:

$$
W_{a d j}(L)=\exp \left(-\sigma_{a d j} R T\right) .
$$

Here $\sigma_{a d j}$ is the QCD string tension in the adjoint representation [7].

With the help of (2.5) it may be seen now that the second term in (2.4) being proportional to Wilson loop also decays exponentially with the increase of the area spanned by loop $L$, and in the case of large distances we simply get

$$
\left.\hat{\rho}\right|_{R T \rightarrow \infty}=N_{c}^{-1} \hat{I} .
$$

Thus the interaction of an arbitrary colour superposition represented by $(2.1,2.3)$ with the QCD stochastic vacuum at large distances (or at big time intervals) leads to an emergence of a fully mixed state with equal probabilities for different colours and without any non-diagonal terms in 
the corresponding density matrix. In the initial basis (consisting of vectors $|1\rangle,|2\rangle,|3\rangle$ ) it would mean the following transition:

$$
\left(\begin{array}{ccc}
|\alpha|^{2} & \alpha \beta^{*} & \alpha \gamma^{*} \\
\alpha^{*} \beta & |\beta|^{2} & \beta \gamma^{*} \\
\alpha^{*} \gamma & \beta^{*} \gamma & |\gamma|^{2}
\end{array}\right) \rightarrow\left(\begin{array}{ccc}
N_{c}^{-1} & 0 & 0 \\
0 & N_{c}^{-1} & 0 \\
0 & 0 & N_{c}^{-1}
\end{array}\right)
$$

Quantum optics provides us with the means to analyze this transition quantitatively. In order to do that we will use the concepts of purity, fidelity and von Neumann entropy.

\subsection{Purity}

Purity $\mathrm{P}$ is defined as $\mathrm{P}=\operatorname{Tr}\left(\hat{\rho}^{2}\right)$ and it represents the closeness of a quantum state to a pure one. For the final state $\hat{\rho}(y)$ depicted by (2.4) we acquire

$$
\mathrm{P}=N_{c}^{-1}+\left(1-N_{c}^{-1}\right) W_{a d j}^{2}(L)
$$

In the absence of the second term (i.e. when $R T \rightarrow \infty$ ) we get $\mathrm{P}=N_{c}^{-1}$ (fully mixed state).

The initial state has a unit purity $\mathrm{P}=\operatorname{Tr}\left(\hat{\rho}_{i n}^{2}\right)=\operatorname{Tr}\left(\hat{\rho}_{i n}\right)=1$ (which obviously means that the state is pure). Note that by taking $R T \rightarrow 0$ and thus $W_{a d j}(L) \rightarrow 1$ in (2.8) (which can be interpreted as absence of interaction with vacuum) we get the very same initial pure state: $\mathrm{P}=1$.

\subsection{Fidelity}

Quantum fidelity is defined as $[18,19]$

$$
F(\omega, \tau)=\operatorname{Tr}(\sqrt{\sqrt{\omega} \tau \sqrt{\omega}})
$$

This quantity represents the square root of the probability of transition between the states described by density matrices $\omega$ and $\tau$. If one of the states is pure, then fidelity is equal to the square root of the overlap between the states [20]. Thus sometimes its definition is squared [21]. In our case, for the final state $\hat{\rho}(y)$ and the initial state $\hat{\rho}_{\text {in }}$ we get

$$
\begin{aligned}
& F\left(\hat{\rho}_{i n}, \hat{\rho}(y)\right)=\operatorname{Tr}\left(\sqrt{\sqrt{\hat{\rho}_{i n}} \hat{\rho}(y) \sqrt{\hat{\rho}_{i n}}}\right)= \\
& \quad=\operatorname{Tr}\left(\hat{\rho}_{i n} \sqrt{N_{c}^{-1}+\left(1-N_{c}^{-1}\right) W_{a d j}(L)}\right) .
\end{aligned}
$$

This time the absence of the second term under the root (when $W_{a d j}(L) \rightarrow 0$ ) returns the value of fidelity $F=N_{c}^{-1 / 2}$. Again we get some non-unit value, which means that the state has changed. And once more it can be seen how $W_{a d j}(L) \rightarrow 1$ gives us the initial state: $F=1$.

It was shown also $([12,16,17])$ that the fidelity may be treated as a quantity which describes stability of quantum motion of the particles. Further considerations obtained under this approach can be found in $[5,17]$. 


\subsection{Von Neumann entropy}

Von Neumann entropy shows how much quantum information is lost in the system during its departure from a pure state [23]. The definition is:

$$
S=-\operatorname{Tr}(\hat{\rho} \ln \hat{\rho}) .
$$

For the initial state we obviously get zero entropy, as the state is pure and the density matrix is idempotent. For the final state in case of $R T \rightarrow \infty$ (see (2.6)) we get:

$$
S=-\operatorname{Tr}\left(N_{c}^{-1} \hat{I} \ln \left(N_{c}^{-1} \hat{I}\right)\right)=\ln N_{c} .
$$

For the intermediate cases of final state (when $R T$ is not large and is not equal to zero) one may rewrite the expression (2.11)

$$
S=\left(1-N_{c}^{-1}\right)\left(1-\ln \left(\frac{\ln W_{a d j}(L)}{N_{c}}\right)+O\left(\ln W_{a d j}(L)\right) .\right.
$$

This expression shows that at distances of order of $1 \mathrm{fm}$ the entropy grows proportionally to the QCD string tension and the string's length, which gives additional weight to the statement that the confinement of quarks can be associated with the loss of quantum information [8].

\section{Squeezed and entangled colour states}

The instability of motion in the confinement region also can be associated with possible phenomena of quantum entanglement and squeezing of color states [24, 25, 26, 27]. Color particles moving through QCD vacuum with large momentum develop quark-gluon jets. Both perturbative and nonperturbative stages of the jet evolution are important. Gluon multiplicity distribution at the end of the perturbative cascade is Poissonian one [28].

Gluon coherent states under the influence of the nonlinearities of QCD Hamiltonian transform into the squeezed and entangled states with sub- (super)-Poissonian multiplicity distributions [25, 26, 27]. Within local parton-hadron duality we can estimate nonperturbative contribution of the gluon squeezed states to the pion correlation functions in the jet narrow ring [29].

The emergence of entagled and squeezed states in QCD becomes possible due to the fourgluon self-interaction, the three-gluon self-interaction does not lead to the effects [25, 26, 27]. Moreover we may amplify or, on the contrary, weaken both the squeezing effect and the system instability using delta-pulses [30]. Two mode gluon squeezed and entangled states with two different colours can lead to quark-antiquark entangled states, role of which could be important for of the confinement and hadronization phenomena [26]. The concept of quantum entanglement was found to be very useful as a model-independent characteristic of the structure of the ground state of quantum field theories which exhibit strong long range correlations, most notably lattice spin systems near the critical points and the corresponding conformal field theories [31]. Quantum entanglement was also considered as an alternative way to probe the confining properties of large- $\mathrm{N}$ gauge theories [32]. Entangled states of pure and mixed multi-quark systems were considered in [10]. It was also shown that in the confinement regime, the entanglement of quark color states is maximal [33]. 


\section{Confinement, critical energy of order-chaos transition and mass of Higgs boson}

The increase in the instability of motion in the confinement region is also connected with the existence of chaotic solutions of Yang-Mills field [6, 34], possible chaos onset [35]. Yang-Mills fields show inherent chaotic dynamics and have chaotic solutions already on classical level [34, 35]. It was shown that Higgs boson and its vacuum quantum fluctuations regularize the system and lead to the emergence of order-chaos transition at some critical energy [36, 37]:

$$
E_{c}=\frac{3 \mu^{4}}{64 \pi^{2}} \exp \left(1-\frac{\lambda}{g^{4}}\right) \text {. }
$$

Here $\mu$ is mass of Higgs boson, $\lambda$ is its self interaction coupling constant, $g$ is the coupling constant of gauge fields. Very important here is the value of mass of Higgs boson. In the region of confinement there exists also the point of order-chaos transition where the fidelity decreased strongly. This connects the properties of stochastic QCD vacuum and Higgs boson mass and coupling constants.

\section{Conclusion}

The stochastic vacuum model proved to be useful and effective when dealing with nonperturbative effects such as the confinement of quarks. Stochastic vacuum of quantum chromodynamics for which only correlators of the second order are important can be considered as environment (in the sense of quantum optics) for color particles (quarks and gluons). The dynamics of YangMills fields, which is inherently chaotic already at the classical level, can be partly regularized by interaction with Higgs fields and by quantum fields fuctuations. Critical point of order-chaos transition appears which corresponds to the point of fidelity exponential decreasing. Squeezinq, entanglement, decoherence and instability accompany nonperturbative evolution of colour particles in QCD vacuum and confinement phenomenon.

It was shown that the interaction colour superposition (representing the quark) with the QCD stochastic vacuum on large scales lead to decoherence and emergence of a fully mixed colour state with equal probabilities for each of the colours.

In this process the initial density matrix of the system undergoes the process of decoherence, so the quantum information contained in its non-diagonal components is being erased and this fact might be associated with the confining properties of QCD vacuum, as the loss of information is proportional to the distance between the colour charges. The measure of the information loss is evaluated using quantum optics' concepts: fidelity, purity and Von Neumann entropy. The calculation was made possible due to the intrinsic qualities of the stochastic vacuum model that allowed to introduce Wilson loop and the according area law. Wilson loop decays exponentially with its decay rate bing equal to the QCD string tension.

\section{References}

[1] J. Ambjørn, P. Olesen. On the Formation of a Color Magnetic Quantum Liquid in QCD. Nuclear Physics B. 170, no. 1, 60-78 (1980). 
[2] A. D. Giacomo, H. Dosch, V. I. Shevchenko, and Y. A. Simonov, Physics Reports 372, no. 4, 319-368 (2002).

[3] D. S. Kuzmenko, Y. A. Simonov, et al. Vacuum, Confinement and QCD Strings in the Vacuum Correlator Method. Uspekhi Fizicheskih Nauk. 174, no. 1 (2004) (in Russian).

[4] Y. A. Simonov. The Confinement. Uspekhi Fizicheskih Nauk. 4 (1996) (in Russian).

[5] V. Kuvshinov. Confinement, Decoherence, Chaos, Higgs boson, Entanglement and so on. Nonlinear Phenomena in Complex Systems. 16, no. 1, 1-6 (2013).

[6] V. Kuvshinov, A. Kuzmin. Gauge Fields and Theory of Deterministic Chaos (Belorussian Science, Minsk, 2006, p. 1-268 in Russian).

[7] V. Kuvshinov, P. Buividovich. White Mixed States in QCD Stochastic Vacuum. Nonlinear Phenomena in Complex Systems. 8, 313-316 (2005).

[8] V. Kuvshinov, P. Buividovich. Decoherence of Quark Colour States in QCD Vacuum. Acta Physica Polonica B. (Proceedings Supplement), 1, no. 3, 579-582 (2008).

[9] V. Kuvshinov, E. Bagashov. Evolution of Colour Superposition in the Stochastic QCD Vacuum. Nonlinear Phenomena in Complex Systems, vol. 16, no. 3 (2013), pp. 242 - 246.

[10] V. Kuvshinov, E. Bagashov. Multi-quark states in the stochastic vacuum. Nonlinear Dynamics and Applications: Proceedings of the Twenty first Annual Seminar NPCS'2014, Minsk, May 20-23, 2014. Vol. 20. P. 32-38.

[11] H. Haken, P. Reineker. The Influence of Exciton Motion on Spin-Resonance. Absorption. Z. Physik. 250, 300 (1972).

[12] P. Reineker. Exciton Dynamics in Molecular Crystals and Aggregates. (Springer-Verlag, Berlin, 1991).

[13] F. Haake. Quantum Signatures of Chaos. (Springer-Verlag, Berlin, 1991).

[14] A. Peres. Quantum Theory: Concepts and Methods. (Kluwer, Dordrecht, 1995).

[15] K. G. Wilson. Confinement of Quarks. Physics Review D. 10, no. 8, 2445-2459 (1974)

[16] V. Kuvshinov, A. Kuzmin. Physics Letters A. 316, 391-394 (2003).

[17] V. I. Kuvshinov, P. V. Buividovich. Fidelity and Wilson Loop for Quarks in Confinement Region. Acta Physica Polonica. 36, no. 2, 195-200 (2005).

[18] A. Uhlmann. The "Transition Probability" in the State Space of a *-Algebra. Rep. Math. Phys. 9, 273 (1976).

[19] M. Hübner. Explicit Computation of the Bures Distance for Density Matrices, Phys. Lett. A, 163, 239-242 (1992).

[20] J. A. Miszczak et al. Sub- and Super-Fidelity as Bounds for Quantum Fidelity, Quantum Information and Computation, 9, no. 1 and 2, 0103-0130 (2009).

[21] R. Jozsa. Fidelity for Mixed Quantum States. J. Mod. Opt. 41 2315-2323 (1994).

[22] A. Gilchrist, N. K. Langford, M. A. Nielsen. Distance Measures to Compare Real and ideal Quantum Processes, Physical Review A, 71, 062310 (2005).

[23] J. von Neumann. Mathematical Foundations of Quantum Mechanics (Princeton University Press, Princeton, 1955). 
[24] V. Kuvshinov, V. Shaporov. Acta Physica Polonica B, 30, no. 59, (1999).

[25] V. Kuvshinov, V. Shaparau. Phys. Atom. Nucl., 65, 309-314 (2002).

[26] V. Kuvshinov, V. Shaparau. Nonlinear Phenomena in Complex Systems, 6, no.4, 898-902 (2003).

[27] V. Kuvshinov, V. Shaparau. Acta Physica Polonica B, 35, no. 1, 443-446 (2004).

[28] S. Lupia, W. Ochs, J. Wosiek. Nucl. Phys. B, 540, 05 (1999).

[29] V. Kuvshinov, V. Shaparau. Nonlinear Dynamics and Applications: Proceedings of the Annual Seminar NPCS'2011, vol. 18, 210 (2011).

[30] V. Kuvshinov, V. Marmish, V. Shaporov. Theor. Math. Phys., 139, 477-490 (2004).

[31] P. Calabrese and J. Cardy. Int.J.Quant.Inf., 4,429 (2006).

[32] L.R. Klebanov, D. Kutasov, A. Murugan. Entanglement as a Probe of Confinement. arXiv:0709.2140v2 [hep-th]

[33] V. Kuvshinov, P. Buividovich. Nonlinear Phenomena in Complex Systems, 13, no. 2, 149-155 (2010).

[34] G. K. Savvidy. Infrared Instability of the Vacuum State of Gauge Theories and Asymptotic Freedom. Physics Letters B. 71, no. 1, 133-134 (1977).

[35] T. Kawabe. Phys. Lett. B, 334, no. 1-2, 127-131 (1994).

[36] V. Kuvshinov, A. Kuzmin. Nonlinear Phenomena in Complex Systems, vol. 2, no.3, 100-104 (1999).

[37] V. Kuvshinov, A. Kuzmin. J. Nonl. Math. Phys. 9, no.4, 382-388 (2002). 